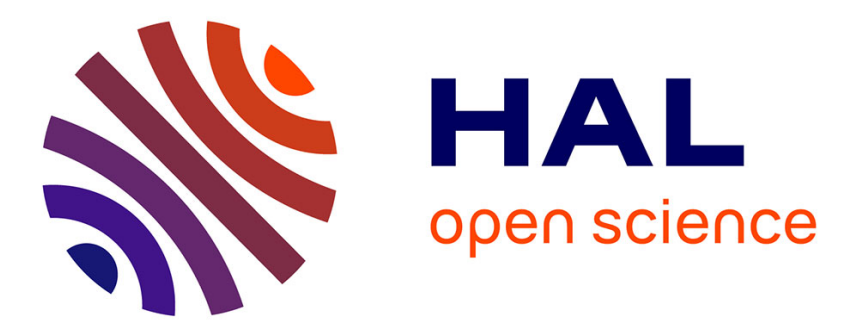

\title{
Frequency-dissymmetric parametric sideband generation in a microstructured fiber
}

Margaux Barbier, Philippe Leproux, Philippe Roy, Philippe Delaye

\section{To cite this version:}

Margaux Barbier, Philippe Leproux, Philippe Roy, Philippe Delaye. Frequency-dissymmetric parametric sideband generation in a microstructured fiber. Journal of the Optical Society of America B, 2013, 30 (11), pp.2889. hal-00879732

\section{HAL Id: hal-00879732 \\ https://hal-iogs.archives-ouvertes.fr/hal-00879732}

Submitted on 4 Nov 2013

HAL is a multi-disciplinary open access archive for the deposit and dissemination of scientific research documents, whether they are published or not. The documents may come from teaching and research institutions in France or abroad, or from public or private research centers.
L'archive ouverte pluridisciplinaire HAL, est destinée au dépôt et à la diffusion de documents scientifiques de niveau recherche, publiés ou non, émanant des établissements d'enseignement et de recherche français ou étrangers, des laboratoires publics ou privés. 


\title{
Frequency-dissymmetric parametric sideband generation in a microstructured fiber
}

\author{
Margaux Barbier, ${ }^{1, *}$ Philippe Leproux, ${ }^{2}$ Philippe Roy, ${ }^{2}$ and Philippe Delaye ${ }^{1}$ \\ ${ }^{1}$ Laboratoire Charles Fabry, Institut d'Optique, CNRS, Univ Paris-Sud, \\ 2 Avenue Augustin Fresnel, 91127 Palaiseau, France \\ ${ }^{2}$ Université de Limoges, XLIM, UMR CNRS 7252, 123 Avenue Albert Thomas, 87060 Limoges, France \\ *Corresponding author: margaux.barbier@institutoptique.fr
}

Received June 26, 2013; revised September 6, 2013; accepted September 6, 2013;

posted September 19, 2013 (Doc. ID 192963); published October 15, 2013

\begin{abstract}
We experimentally demonstrate the nonlinear generation of frequency-dissymmetric sidebands by injecting picosecond pump pulses inside the fundamental mode of a silica-core photonic crystal fiber in its normal dispersion regime. A systematic analysis highlights the fact that this phenomenon is based on the combination of the two major nonlinear effects occurring inside the fiber: self-phase modulation and degenerate four-wave mixing. (c) 2013 Optical Society of America
\end{abstract}

OCIS codes: $\quad$ (190.4370) Nonlinear optics, fibers; (190.4380) Nonlinear optics, four-wave mixing; (190.3270)

Kerr effect; (060.5295) Photonic crystal fibers.

http://dx.doi.org/10.1364/JOSAB.30.002889

\section{INTRODUCTION}

Four-wave mixing (FWM) in optical fibers [1] is the basis of a large variety of applications. This mechanism has been demonstrated as one of the sources for supercontinuum generation [2]. On the other hand, fiber-optic parametric amplifiers (FOPAs) can be used for signal amplification and wavelength conversion, even in the visible range of the optical spectrum [3]. FOPAs can also be employed for ultrafast signal processing, due to the quasi-instantaneous nature of this nonlinear process [4]. Moreover, spontaneous FWM in optical fibers $[\underline{5}, 6]$ opens the way to all-fiber quantum communication devices useful in the field of quantum communication networks [7]].

The development of photonic crystal fibers (PCFs) [8] led to a leap forward in the field of nonlinear optics in fibers. Indeed, the original properties of such fibers allow us to control nonlinear effects and to reinforce their efficiency; that is to say, to optimize the performances of devices that are based on these nonlinear effects [9-13]. For example, efficient FWM occurs only when the phase-matching condition is satisfied; thus pump, signal, and idler wavelengths must be close to the zero-dispersion wavelength $\lambda_{0}$ of the fiber. In the case of conventional silica fibers, $\lambda_{0}$ is about $1.3 \mu \mathrm{m}$, and generated photons necessarily have wavelengths close to this value, thus covering the telecoms wavelength bands. PCFs allow us to overcome this limitation: appropriately designing the microstructuration of the fiber enables to tailor its dispersion properties and, particularly, to position the value of $\lambda_{0}$ in the near infrared, opening the possibility to generate pairs of photons in the visible [6].

In pulsed regime, FWM is generally associated to another well-known nonlinear effect, governed by the same nonlinear parameter: self-phase modulation (SPM), which occurs when a single pulsed pump beam is injected in the silica core of a fiber [14], and that is responsible for a spectral broadening of the pump beam. These phenomena are usually considered as decoupled from each other in FWM experiments and treated independently in analytical models. For example, if supercontinuum generation is mainly governed by SPM in the fs regime, it is the FWM mechanism that governs supercontinuum generation in the ps regime; in that case, it was shown that simple CW FWM equations are sufficient to describe the observations [2]. Identically, for the generation of pairs of photons [5-7], where ps laser sources are usually used, the $\mathrm{CW}$ modeling of FWM is generally successfully used to describe the experimental results. In this paper, we demonstrate that, using a $\mathrm{PCF}$ in its normal dispersion regime, it is possible to reach an operating point for which such a decoupling is no longer valid. Independently describing spontaneous FWM, on the one hand, and SPM on the other hand, leads to simulation results that are not consistent with our experimental observations. Indeed, as we will see in Section $\underline{4}$, we actually observe for the first time to our knowledge a spontaneous FWM phenomenon when injecting pump pulses at a wavelength $\lambda_{\text {inj }}$ for which the calculated phase-matching curve predicts that no efficient FWM process can occur. Moreover, this FWM phenomenon shows a clear frequency dissymmetry with respect to the injected pulse frequency.

The paper is organized as follows. Section $\underline{2}$ starts with a brief theoretical description of the two nonlinear processes involved in our experiments: SPM and degenerate FWM. In Section 3, we describe the properties of our PCF and the experimental setup. In Section $\underline{4}$, we present our experimental results and discuss the role of SPM in getting efficient frequency-dissymmetric FWM in the normal dispersion regime of a fiber. Finally, a conclusion is drawn in Section $\underline{5}$.

\section{THEORETICAL BACKGROUND}

In this section, we give a brief theoretical description of the two nonlinear phenomena involved in our experiments: SPM 
and degenerate FWM. These processes are both governed by the real part of the third-order nonlinear susceptibility $\chi^{(3)}$ of the medium.

For the experiments presented in this paper, the slowly varying envelope and the quasi-monochromatic field approximations can be adopted, and all the equations presented in this section are valid in this framework. However, since the propagation duration is high (approximately $5 \mathrm{~ns}$ in a $1 \mathrm{~m}$ long fiber) compared to the pulse duration we used (about $1 \mathrm{ps}$ ), the effects of the group-velocity mismatch (GVM) should be taken into account, particularly regarding the FWM process. Note that we can use a scalar approach, since all the involved fields in our experiments present the same linear polarization state (see Section 3 ).

\section{A. Self-Phase Modulation}

SPM originates from the intensity-dependence of the refractive index in nonlinear optical media. It results in a spectral broadening of optical pulses injected inside the medium, without any temporal change [14].

Solving the nonlinear propagation equation with negligible dispersion shows that SPM gives rise to an intensitydependent phase-shift, which reaches its maximum value at the maximum of the pulse temporal shape. This maximum value is

$$
\Phi_{\mathrm{NL}, \max }(z)=\gamma P_{p} L_{\mathrm{eff}}(z)=\gamma P_{p} \frac{1-e^{-\alpha z}}{\alpha}
$$

where $P_{p}$ is the injected pump peak power, $\alpha$ is the attenuation constant (due to linear losses), and $L_{\text {eff }}(z)$ is the effective propagation length of the pulse inside the nonlinear medium. $\gamma$ is the nonlinear coefficient of the medium, given by [14]

$$
\gamma=\frac{\omega_{p} n_{2}^{(I)}}{c A_{\mathrm{eff}}},
$$

where $c$ is the speed of light in vacuum, $\omega_{p}$ is the pump frequency, $n_{2}^{(I)}$ is the nonlinear refractive index of the material (in our case, the propagating mode is mainly contained in the silica core of a fiber, and thus we can use the $n_{2}^{(I)}$ of silica: $\left.2.7 \times 10^{-20} \mathrm{~m}^{2} \cdot \mathrm{W}^{-1}[14]\right)$, which is proportional to the real part of $\chi^{(3)}$, and $A_{\text {eff }}$ is the effective mode area.

Intensity dependence of the nonlinear phase shift results in the creation of new spectral components, due to the fact that the optical frequency is no longer constant in the total temporal width of the pulse: the pulse is chirped. The instantaneous optical frequency differs from the central frequency, and the difference $\delta \omega$ is given by

$$
\delta \omega(t)=-\frac{\partial \Phi_{\mathrm{NL}}(t)}{\partial t}
$$

Thus, the initial spectrum is broadened, and after a propagation length $z$ inside the nonlinear medium, this spectral broadening is proportional to the maximum nonlinear phase shift [14]:

$$
\frac{\Delta \lambda(z)}{\Delta \lambda(z=0)} \propto \Phi_{\mathrm{NL}, \max }(z)
$$

The experiments presented in this paper involve hyperbolic secant pulses [see Fig. 1(a)]. For such a temporal shape, an
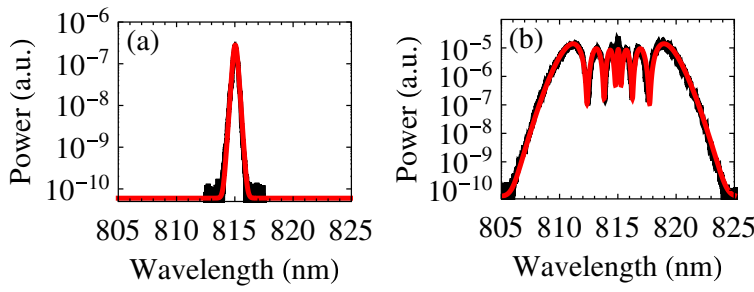

Fig. 1. Measured (black curves) intensity spectra (in logarithmic scale) for an hyperbolic secant pulse (with an injected peak power equal to $407 \mathrm{~W}$ ) at the input (a) and at the output (b) of a third-order nonlinear medium, with the simulated (red) spectra [see Eq. (5)] using parameters $\alpha=0.17 \mathrm{~m}^{-1}, T_{0}=1.1 \mathrm{ps}, \Phi_{\mathrm{NL}, \max }=6 \pi$, and $z=0.97 \mathrm{~m}$ and a central wavelength equal to $815 \mathrm{~nm}$.

analytical model giving the spectral evolution due to SPM [15] [see Fig. 1(b)] is available. According to this analytical model, if we call $U(t ; z=0)=\operatorname{sech}\left(t / T_{0}\right)$ the normalized amplitude of the injected pulse, the intensity spectrum after a propagation length $z$ is given by

$$
\begin{aligned}
I(\omega ; z)= & I_{0} e^{-\alpha z} T_{0}^{2} \operatorname{sech}^{2}\left(\frac{\pi \omega T_{0}}{2}\right) \\
& \times{ }_{2} F_{2}\left(\left\{\frac{1}{2}+i \frac{\omega T_{0}}{2}, \frac{1}{2}-i \frac{\omega T_{0}}{2}\right\},\left\{\frac{1}{2}, 1\right\}, i \Phi_{\mathrm{NL}, \max }(z)\right) \\
& \times{ }_{2} F_{2}\left(\left\{\frac{1}{2}-i \frac{\omega T_{0}}{2}, \frac{1}{2}+i \frac{\omega T_{0}}{2}\right\},\left\{\frac{1}{2}, 1\right\},-i \Phi_{\mathrm{NL}, \max }(z)\right),
\end{aligned}
$$

where ${ }_{2} F_{2}\left(\left\{a_{1}, a_{2}\right\},\left\{b_{1}, b_{2}\right\}, x\right)$ is the generalized hypergeometric function with coefficients $\{2 ; 2\}$.

\section{B. Degenerate Four-Wave Mixing}

In degenerate FWM, pump photons at frequency $\omega_{p}$ are annihilated in pairs to simultaneously create a pair of signal and idler photons at frequencies $\omega_{s}$ and $\omega_{i}$, respectively, such as $\omega_{p}-\omega_{s}=\omega_{i}-\omega_{p}$ (energy conservation, which implies that the signal and idler photons are generated symmetrically in frequency with respect to the pump spectrum) [14]. This process occurs with high efficiency only if the phase-matching condition (i.e., momentum conservation) is satisfied. The phase-matching condition depends on the values of the propagation constant $\beta$ at frequencies $\omega_{p}, \omega_{s}$, and $\omega_{i}$, the nonlinear coefficient $\gamma$ and the pump peak power $P_{p}$ and can be written as $[14]$

$$
\beta\left(\omega_{s}\right)+\beta\left(\omega_{i}\right)-2 \beta\left(\omega_{p}\right)+2 \gamma P_{p}=0 .
$$

It is easy to show that this condition, which allows us to calculate the phase-matching curve of the fiber for degenerate FWM (for a given pump power), can be simplified to a condition on the group-velocity dispersion (GVD) $\beta_{2}(\omega)$ (second derivative of $\beta$ with respect to $\omega$ ) of the fiber, which can be experimentally determined (by a time-of-flight measurement, for example).

In amplified spontaneous degenerate FWM with pump photons at $\omega_{p}$, the amplification gain at frequency $\omega$ is given by [14]

$$
G\left(\omega_{p} ; \omega\right)=\left(\frac{\gamma P_{p}}{g\left(\omega_{p} ; \omega\right)}\right)^{2} \sinh ^{2}\left(g\left(\omega_{p} ; \omega\right) L_{\mathrm{FWM}}\right),
$$


where $L_{\mathrm{FWM}}$ is the interaction length for FWM, and $g\left(\omega_{p} ; \omega\right)$ is the parametric gain given by

$$
g\left(\omega_{p} ; \omega\right)=\sqrt{\frac{\Delta k\left(\omega_{p} ; \omega\right)}{2}\left(2 \gamma P_{p}-\frac{\Delta k\left(\omega_{p} ; \omega\right)}{2}\right)}
$$

with

$$
\Delta k\left(\omega_{p} ; \omega\right)=2 \beta\left(\omega_{p}\right)-\beta(\omega)-\beta\left(2 \omega_{p}-\omega\right) .
$$

When the phase-matching condition is perfectly satisfied $\left(\Delta k\left(\omega_{p} ; \omega\right)=2 \gamma P_{p}\right)$, the parametric gain reaches its maximum value $g_{\max }\left(\omega_{p}\right)=\gamma P_{p}$, and the amplification gain becomes

$$
G_{\mathrm{max}}\left(\omega_{p} ; \omega_{s}\right)=\sinh ^{2}\left(\gamma P_{p} L_{\mathrm{FWM}}\right)=\sinh ^{2}\left(\Phi_{\mathrm{NL}, \max } \frac{L_{\mathrm{FWM}}}{L_{\mathrm{eff}}}\right),
$$

where $\Phi_{\mathrm{NL}, \max }$ and $L_{\text {eff }}$ are the values of the maximum nonlinear phase shift and the effective propagation length [see Eq. (1)] at the output of the fiber, i.e., with $z=L_{\text {fiber }}$ (physical length of the fiber).

The interaction length $L_{\mathrm{FwM}}$ can be lower than the effective length of the fiber (which is already lower than the physical length of the fiber because of linear losses) due to temporal walk-off, which can occur between the pump pulses and the generated signal and idler pulses.

Interestingly, Eq. (10) underlines the fact that FWM and SPM are governed by the same parameter: the maximum nonlinear phase-shift $\Phi_{\mathrm{NL}, \max }$ or, in other words, the nonlinear coefficient $\gamma$, i.e. the nonlinear refractive index $n_{2}^{(I)}$ (which is proportional to the real part of $\left.\chi^{(3)}\right)$.

Experiments that are presented in this paper involve highgain regime. In such a regime, we can use a classical approach to describe amplified spontaneous FWM, which leads us to show that the expression of the generated power on a frequency range $[\omega-(\Delta \omega / 2) ; \omega+(\Delta \omega / 2)]$ when pumping at $\omega_{p}$ is $[\underline{16}]$

$$
P\left(\omega_{p} ; \omega ; \Delta \omega\right)=\frac{h}{4 \pi^{2}} \int_{\omega-\frac{\Delta \omega}{2}}^{\omega+\frac{\Delta \omega}{2}}\left(G\left(\omega_{p} ; \omega^{\prime}\right)-\frac{1}{2}\right) \omega^{\prime} \mathrm{d} \omega^{\prime} .
$$

Note that if $\Delta \omega$ (which, in practice, represents the spectral resolution of the OSA used to measure the spectra) is small enough (that is to say, smaller than the spectral width of the generated parametric bands), we can write

$$
P\left(\omega_{p} ; \omega ; \Delta \omega\right) \sim \frac{h}{4 \pi^{2}} \omega \Delta \omega\left(G\left(\omega_{p} ; \omega\right)-\frac{1}{2}\right),
$$

and thus, when the phase-matching condition is perfectly satisfied (i.e., at the maximum value of the amplification gain), we get [according to Eq. (10) and in the high gain limit]

$$
P_{\max }\left(\omega_{p} ; \omega_{s} ; \Delta \omega\right) \sim \frac{h}{4 \pi^{2}} \omega_{s} \Delta \omega \sinh ^{2}\left(\Phi_{\mathrm{NL}, \max } \frac{L_{\mathrm{FWM}}}{L_{\mathrm{eff}}}\right) .
$$

\section{Two Usually Decoupled Phenomena}

As we already said, FWM and SPM are governed by the same parameter (the real part of $\chi^{(3)}$ ). However, they are usually decoupled. Indeed, in silica, even after SPM-induced spectral broadening, the pump spectral width usually remains lower than the parametric gain spectral width, on the one hand, and lower than the spectral shift between the pump and FWM-generated signal and idler waves, on the other. Thus SPM-induced spectral broadening of the pump usually has no influence on parametric generation by FWM.

In this paper, we demonstrate that we can find a regime in which this decoupling between FWM and SPM-induced spectral broadening is not valid anymore, and that the latter plays a key role in getting a frequency-dissymmetric FWM.

\section{EXPERIMENTAL SETUP}

\section{A. Photonic Crystal Fiber Properties}

The silica-core PCF used in our experiments (XLIM, RTI-1605UV, see inset of Fig. 2) exhibits a very small (but nonzero) birefringence $\left(\Delta n<\overline{10} 0^{-5}\right)$, thus giving rise to two principal axes.

The GVD $\beta_{2}(\omega)$ of this PCF has been experimentally determined by an interferometric time-of-flight measurement [17] for wavelengths from 740 to $930 \mathrm{~nm}$ on each of the two principal axes. Due to the very small birefringence, the results are quasi-identical for the two axes. For the principal axis used in the following experiments, a polynomial fit to our experimental data leads to

$$
\beta_{2}(\omega)=\left(K_{0}+K_{1} \omega+K_{2} \omega^{2}+K_{3} \omega^{3}\right),
$$

with $\beta_{2}(\omega)$ in $\mathrm{s}^{2} \cdot \mathrm{m}^{-1}, \omega$ in $\mathrm{rad} \cdot \mathrm{s}^{-1}$ and polynomial fit parameter values given by:

$$
\begin{aligned}
& K_{0}=-1.8110500896234425 \cdot 10^{-24}, \\
& K_{1}=2.1530389065354214 \cdot 10^{-39}, \\
& K_{2}=-8.706787346496974 \cdot 10^{-55}, \\
& K_{3}=1.2061182225010229 \cdot 10^{-70} .
\end{aligned}
$$

The result is consistent with the analytical calculation provided by Saitoh and Koshiba [18] using the geometrical parameters of our PCF (a hole diameter $d=1.7 \mu \mathrm{m}$ and a hole pitch

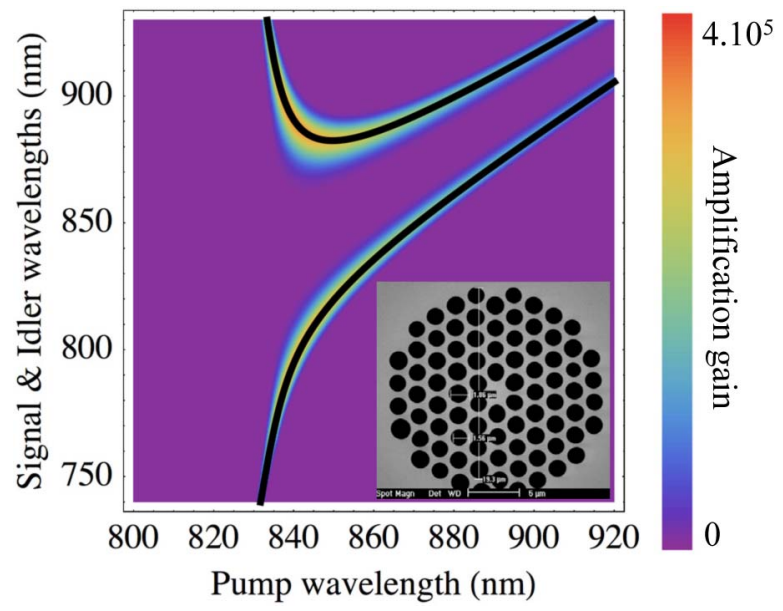

Fig. 2. Calculated phase-matching curve (black) and amplification gain profile based on the GVD measurement (plotted with a pump peak power value of $200 \mathrm{~W}$ ). Inset: scanning electronic microscopy (SEM) image of the transverse structure of our silica-core PCF. 
Table 1. Properties of Our Silica-Core PCF

\begin{tabular}{lccc}
\hline Property & Notation & Value & Unit \\
\hline Length & $L_{\text {fiber }}$ & 0.97 & $\mathrm{~m}$ \\
Attenuation & $\alpha$ & 0.17 & $\mathrm{~m}^{-1}$ \\
Effective length & $L_{\text {eff }}$ & 0.89 & $\mathrm{~m}$ \\
Birefringence & $\Delta n$ & $<10^{-5}$ & \\
Hole diameter & $d$ & 1.7 & $\mu \mathrm{m}$ \\
Hole pitch & $\Lambda$ & 2.3 & $\mu \mathrm{m}$ \\
Effective area & $A_{\text {eff }}$ & 4.0 & $\mu \mathrm{m}^{2}$ \\
Nonlinear index & $n_{2}^{(I)}$ & $2.7 \times 10^{-20}$ & $\mathrm{~m}^{2} \cdot \mathrm{W}^{-1}$ \\
Nonlinear coefficient & $\gamma$ & $\sim 0.05$ & $\mathrm{~m}^{-1} \cdot \mathrm{W}^{-1}$ \\
Zero-dispersion wavelength & $\lambda_{0}$ & 834 & $\mathrm{~nm}$ \\
Vertical asymptote & & $\sim 830$ & $\mathrm{~nm}$ \\
\hline
\end{tabular}

$\Lambda=2.3 \mu \mathrm{m}$, estimated with a precision of about $5 \%$ on the SEM image of the transverse structure of the PCF, see inset of Fig. 2). This analytical model allows us to calculate the effective $V$ parameter of our PCF, and then, using the effective mode area expression as a function of this effective $V$ parameter [19], we find $A_{\text {eff }}=4.0 \mu \mathrm{m}^{2}$. And thus, according to Eq. (2), for a pump wavelength around $830 \mathrm{~nm}$, the nonlinear coefficient of our silica-core PCF is about $0.05 \mathrm{~m}^{-1} \cdot \mathrm{W}^{-1}$.

Finally, from this GVD measurement, we estimate the zerodispersion wavelength of the PCF: $\lambda_{0}=834 \mathrm{~nm}$, and we deduce the phase-matching curve given in Fig. 2 , in which we note that there is a vertical asymptote around $830 \mathrm{~nm}$. Thus FWM should not be observed if the pump wavelength $\lambda_{p}$ is lower than this limit value.

The various properties of our silica-core PCF are summarized in Table 1.

\section{B. Setup}

Our experimental setup is shown schematically in Fig. 3.

A Ti:sapphire laser delivers picosecond hyperbolic secant pulses [see Fig. 1(a)] with an $80 \mathrm{MHz}$ repetition rate. The pulse duration $T_{0}$ is systematically measured using an autocorrelator.

The pump beam is linearly polarized, and we use a halfwave plate (HWP) to choose the orientation of this linear polarization state. As we already said, the PCF presents a residual birefringence that gives rise to two principal axes. We align the polarization state of the pump beam with one of these principal axes, and thus we get a linearly polarized beam at the output of the PCF. Even if this paper exclusively provides the results that have been obtained through a systematic analysis on this principal axis, it is important to note that similar results were obtained on both axes.

We inject the pump pulses through a microscope objective. At the output of the PCF, we use an imaging lens and a video camera to monitor the transverse profile of the beam. Thus we can check that the injected beam propagates on the fundamental mode of the PCF. Thanks to a removable mirror,

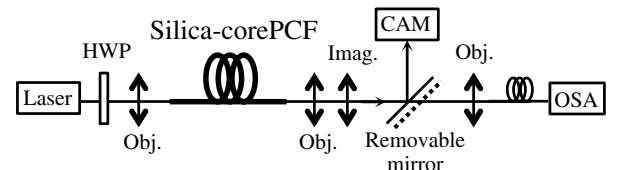

Fig. 3. Experimental setup used to observe frequency-dissymmetric FWM in our PCF. (Obj., microscope objective; HWP, half-wave plate; Imag., imaging lens.) we can choose between viewing the transverse profile of the beam and injecting it into an optical spectrum analyzer (OSA) in order to measure the output spectrum. Note that the shape of the output spectrum also gives information about the injection quality: as soon as the injected beam propagates on more than one transverse mode, the measured output spectrum becomes unstable and oscillating. In other words, the quality and stability of the output spectrum guarantees that the beam is injected on the fundamental mode of the fiber.

Moreover, the output spectrum measurement also allows us to check that the parametric sidebands are linearly copolarized with the pump beam. Thus the scalar approach used in Section $\underline{2}$ is actually valid.

\section{Getting the Pump Power Value by Measuring the SPM-Induced Spectral Broadening of the Pump}

In all our experiments, precise knowledge of the value of the injected peak power $P_{p}$ is essential, since it drives the spectral location and the power level of the generated sidebands. Thus we need an experimental way to accurately measure this parameter. That is why the analytical model resulting in Eq. (5) is useful.

Indeed, by fitting this analytical model to the SPM-broadened spectrum measured at the output of the PCF, we can get the value of $\Phi_{\mathrm{NL}, \max }$ (maximum nonlinear phase shift at the output of the fiber), which is the only adjustable parameter in Eq. (5) [we have prior knowledge of the propagation length $L_{\text {fiber }}$, the attenuation constant $\alpha$ (see Table 1 ), and the pulse duration $T_{0}$ by an autocorrelation measurement]. Thus we can accurately deduce the value of $P_{p}$ according to Eq. (1).

\section{RESULTS AND DISCUSSION}

On the phase-matching curve (see Fig. 2), the vertical asymptote at $\lambda_{p} \sim 830 \mathrm{~nm}$ reveals that FWM should not be observed if the pump wavelength $\lambda_{p}$ is lower than this limit value.

Our experimental observations are different from this prediction. Indeed, we observe, for example, that an amplified spontaneous FWM process actually occurs when injecting pulses at $815 \mathrm{~nm}$, with generation of signal and idler spectral bands around 925 and $745 \mathrm{~nm}$, respectively (see Fig. 4), contrary to what is expected according to the phase-matching curve. Moreover, a clear frequency dissymmetry with respect to the injected pulse frequency $\left(\omega_{\text {inj }}=2 c / \lambda_{\text {inj }}\right)$ is seen: generated signal and idler frequencies $\omega_{s}$ and $\omega_{i}$ do not satisfy

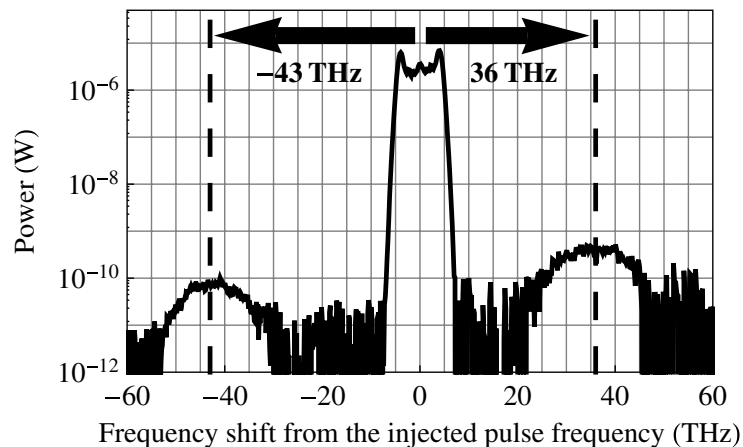

Fig. 4. Example of frequency-dissymmetric spectrum, which has been observed when injecting picosecond hyperbolic secant pulses at $815 \mathrm{~nm}$ inside the fundamental mode of the PCF (OSA resolution: $2 \mathrm{~nm}$, i.e., $0.9 \mathrm{THz}$ ). The spectral width of the injected beam is increased by a factor of about 100 due to SPM 
$\omega_{s}+\omega_{i}=2 \omega_{\text {inj. }}$. Of course, this result does not mean that the energy conservation is not satisfied, but that the signal and idler photon pairs are not created from two photons at frequency $\omega_{\text {inj }}$ but at a slightly different frequency $\omega_{\text {source }}$ such as $2 \omega_{\text {source }}=\omega_{s}+\omega_{i}$.

To understand the origin of this phenomenon, we made some spectrum measurements varying the injected beam wavelength on the one hand, and the injected peak power on the other. The results clearly highlight the key role played by SPM-induced spectral broadening in getting an efficient frequency-dissymmetric FWM process in the normal dispersion regime of our PCF.

\section{A. Injected Wavelength Influence}

When varying the injected wavelength $\lambda_{\text {inj }}$, three operating regimes clearly appear (see Fig. 5):

(1) The first one (zone I), which corresponds to the values of $\lambda_{\text {inj }}$ much higher than $\lambda_{0}$ (anomalous dispersion regime), and for which our experimental results (generated sideband maxima) follow the phase-matching curve, and the FWM process is frequency-symmetric as expected.

(2) The second one (zone II.a), which corresponds to the values of $\lambda_{\text {inj }}$ much lower than $\lambda_{0}$ (normal dispersion regime), and for which we observe an unexpected and frequencydissymmetric efficient FWM process.

(3) A transition regime between these two first regimes (zone II.b), for which we can see that the generated sidebands exhibit two local maxima each (see inset of Fig. 5). The frequency dissymmetry is exhibited for both pairs of maxima (the external one, the farther from the pump spectrum; and the internal one, the closer from the pump spectrum), as it can be clearly seen in Fig. $\underline{6}$. The origin of such doublemaximum sidebands is discussed in Subsection 4.C.

Figure $\underline{6}$ presents the evolution of $\lambda_{\text {source }}$ as a function of $\lambda_{\text {inj }}$, clearly showing the dissymmetry that appears when moving to the normal dispersion regime. Recall that $\lambda_{\text {source }}$ is simply calculated thanks to the energy-conservation condition, based on wavelengths $\lambda_{s}$ and $\lambda_{i}$ measured at the maxima of the

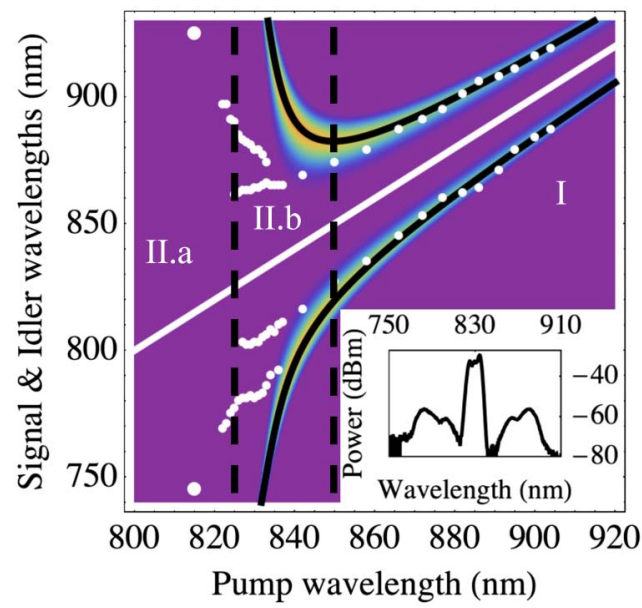

Fig. 5. Comparison between the experimental location of the generated sidebands (for a quasi-constant injected pump peak power of about $200 \mathrm{~W}$ ) with the phase-matching curve. The white straight line $(y=x)$ corresponds to the value of $\lambda_{\text {inj. }}$. Inset: typical spectrum observed in zone II.b, with double-maximum sidebands.

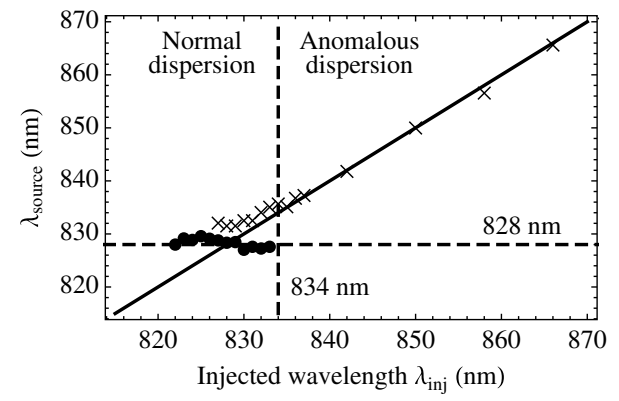

Fig. 6. Evolution of $\lambda_{\text {source }}$ as a function of $\lambda_{\text {inj. }}$. Crosses: anomalous dispersion regime and internal sideband (in the transition regime) results. Dots: external sideband (in the transition regime) results. Straight line $(y=x)$ : value of $\lambda_{\text {inj }}$. For values of $\lambda_{\text {inj }}$ higher than $870 \mathrm{~nm}, \lambda_{\text {source }}$ remains equal to $\lambda_{\text {inj }}$ (the corresponding crosses, which are not plotted here, are still on the straight line).

generated sidebands. We clearly see that $\lambda_{\text {source }}$ is equal to $\lambda_{\text {inj }}$ in the anomalous dispersion regime, as expected. But the dissymmetry is well viewed in the normal dispersion regime since $\lambda_{\text {source }}$ is not equal to $\lambda_{\text {inj }}$ anymore.

Moreover, the dashed straight line, corresponding to $\lambda_{\text {source }}=\lambda_{\text {lim }}=828 \mathrm{~nm}$, shows that $\lambda_{\text {source }}$ is always higher than this limit value $\lambda_{\lim }$. In other words, it highlights the fact that only wavelengths higher than $\lambda_{\text {lim }}$ typically can effectively be used as pump for an efficient FWM process. As this limit value seems to correspond to the vertical asymptote (located around $830 \mathrm{~nm}$ ), this result is consistent with the phasematching curve.

Another interesting point, which is well viewed in Figs. $\underline{5}$ and 6 , is the continuity exhibited by the internal and external maximum pairs of zone II.b with, respectively, the sidebands of zone I and the sidebands of zone II.a. This spectral location continuity is added to a power-level continuity, shown in Fig. 7. Indeed, the internal maximum power level is different from the external maximum-power level, and this difference evolves with $\lambda_{\text {inj. }}$. More precisely, the internal maxima, which follow on from the sidebands of zone I, are predominant as long as $\lambda_{\text {inj }}>832 \mathrm{~nm}$ typically. Their power level decreases, however, as $\lambda_{\text {inj }}$ decreases, whereas the power level of the external maxima grows progressively. The internal maxima end up disappearing when $\lambda_{\text {inj }}$ becomes lower than $824 \mathrm{~nm}$ typically. Only the external maxima remain observable, which are in the continuity of the sidebands of zone II.a.

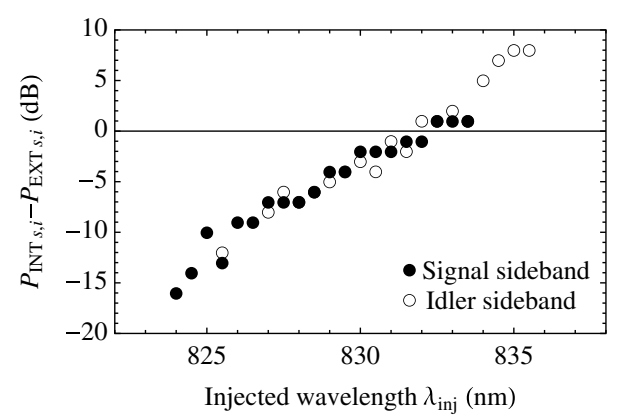

Fig. 7. Difference between internal and external maximum power level (in $\mathrm{dB}$ ) as a function of the injected wavelength. For injected wavelengths higher than $834 \mathrm{~nm}$ (resp. lower than $825 \mathrm{~nm}$ ) typically, the power difference between the two maxima can be evaluated with good reliability only on the idler (resp. signal) sideband; that is why only idler (resp. signal) data is plotted. 


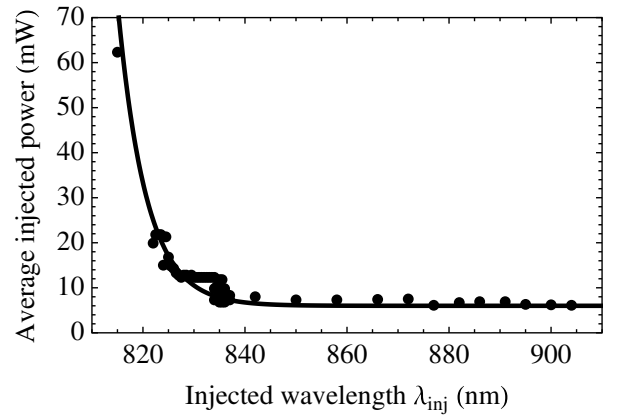

Fig. 8. Average injected power used for the spectrum measurements presented in this subsection. Our experimental criterion was to keep, on the OSA, approximately the same sideband power level for all the measured spectra. This experimental data interestingly highlights a trend showing that the injected power has to be increased when $\lambda_{\text {inj }}$ decreases from $\lambda_{0}$. The solid line is a guideline.

This phenomenon is a clear signature of a competition between two mechanisms responsible for the presence of the two maxima.

For this set of experiments, a last interesting point remains. Indeed, we noted that the minimum peak power that we had to inject inside the PCF in order to observe the parametric sideband generation became higher and higher when we entered more and more heavily in the normal dispersion regime (see Fig. 8). This result is far from insignificant, since it actually allows us to intuitively understand the key role played by SPM in getting a frequency-dissymmetric FWM process.

Indeed, at the input of the fiber, the spectral width of the injected pulses is very small (typically less than $0.5 \mathrm{~nm}$ ), and if the injected wavelength is lower than $830 \mathrm{~nm}$ typically (vertical asymptote location), according to the phase-matching curve, no efficient FWM should be observed. But, during the propagation inside the fiber, the spectrum broadens due to SPM, and if the injected peak power is high enough to produce a sufficiently large broadening factor (see Subsection 2.B), a part of the spectrum can cross beyond the vertical asymptote and effectively be used as pump for an efficient FWM process. Of course, the lower $\lambda_{\text {inj }}$ is, the larger the broadening factor has to be in order to make a part of the injected spectrum cross beyond the vertical asymptote and intercept the phase-matching curve.

Thus, understanding this phenomenon as a FWM process assisted by SPM-induced spectral broadening is consistent with all the experimental results presented in this subsection. Moreover, it has been quantitatively confirmed by our second set of experiments by varying the injected peak power (see Subsection $\underline{4 . B}$ below).

\section{B. Injected Power Influence}

For these experiments, we chose three characteristic wavelengths, one for each operating regime described in the previous subsection: $873 \mathrm{~nm}$ (zone I); $830 \mathrm{~nm}$ (zone II.b); and $825 \mathrm{~nm}$ (zone II.a). For each of them, we measured the output spectrum by varying the injected peak power (see Fig. 9).

We know that, when the phase-matching condition is perfectly satisfied, the amplification gain increases exponentially with the pump peak power and the interaction length $L_{\mathrm{FWM}}$ [see Eq. (10)]. In other words, by plotting the generated sideband maximum-power level $\left(P_{s, i}\right.$, expressed in $\left.\mathrm{dBm}\right)$ as a function of the injected peak power $\left(P_{\mathrm{inj}}\right.$, expressed in $\left.\mathrm{W}\right)$,
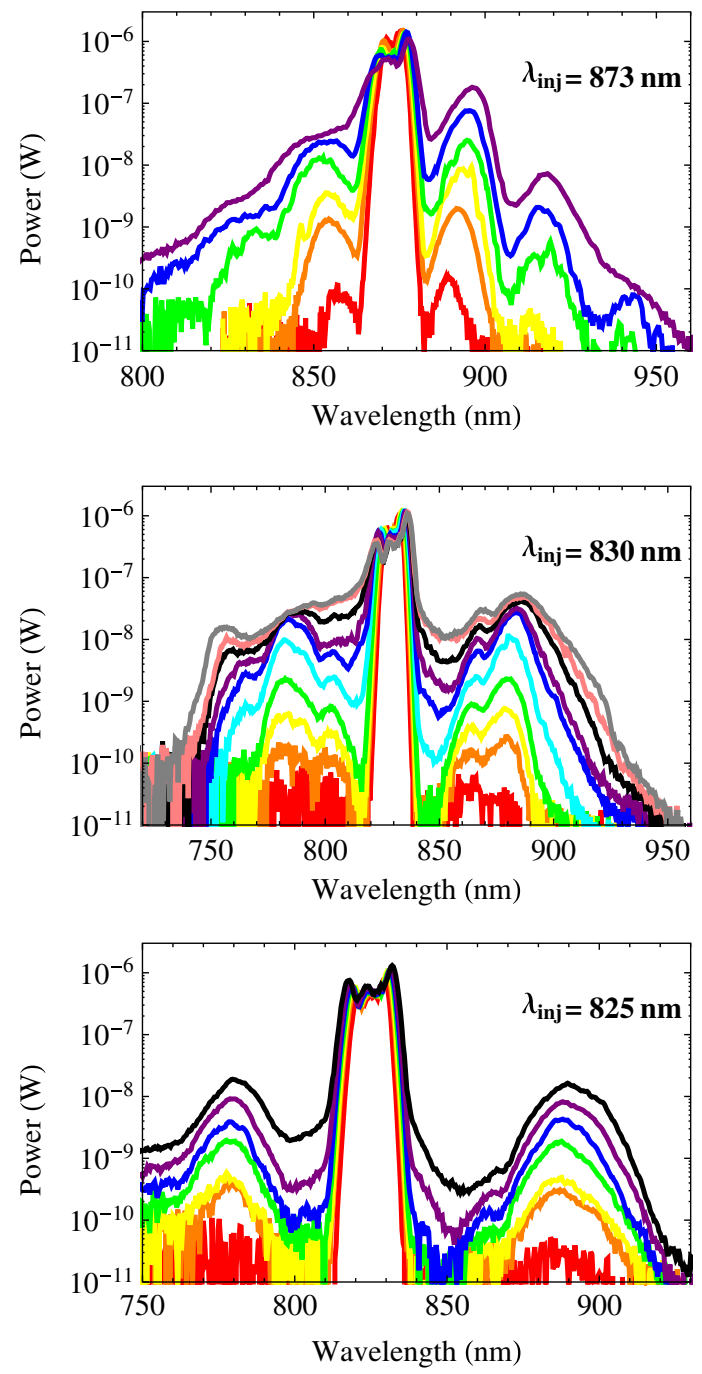

Fig. 9. Measured spectrum networks for $\lambda_{\text {inj }}=\{873 ; 830 ; 825\} \mathrm{nm}$ and $P_{\text {inj }}$ varying typically between 170 and $290 \mathrm{~W}$ for $\lambda_{\text {inj }}=873 \mathrm{~nm}$, between 230 and $510 \mathrm{~W}$ for $\lambda_{\text {inj }}=830 \mathrm{~nm}$, and between 330 and $530 \mathrm{~W}$ for $\lambda_{\text {inj }}=825 \mathrm{~nm}$ (OSA resolution: $2 \mathrm{~nm}$ ). For $\lambda_{\text {inj }}=873 \mathrm{~nm}$, we see the apparition of a cascade FWM process when $P_{\text {inj }}$ is above $230 \mathrm{~W}$ typically.

we must find a straight line, whose slope is proportional to $L_{\text {FWM }}$ and (in first approximation) independent of the wavelength.

As we can see in Fig. 10, we indeed find a straight line for experimental data in the anomalous dispersion regime (we can take this straight line as a reference, since we know that everything works as expected in this regime). Moreover, by superimposing the experimental spectra with the simulation results provided by the model described in Subsection 2.A [see Eqs. (7) and (11)], we can see that this simple model is quite consistent with the experimental observations (see Fig. 11), at least as long as the nonlinear effects remain quite weak (for high injected peak power values, the output spectrum explodes with the apparition of a cascade FWM process, and a simple description of the involved nonlinear processes becomes impossible). Indeed, this simple model gives:

(1) The spectral location of the sideband maxima with only a 3- or 4-nm-shift compared to the experimental results. 


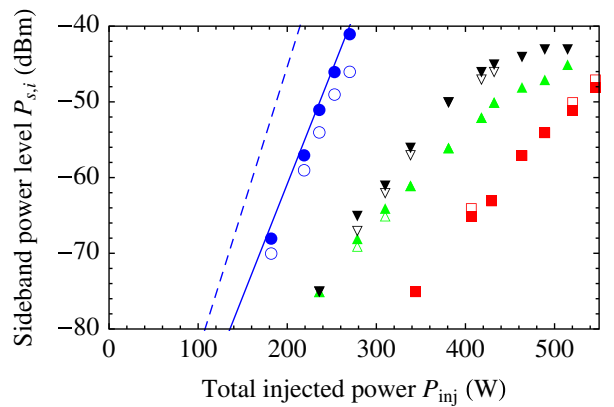

Fig. 10. Measured sideband power level (from spectra in Fig. 9) plotted as a function of the total injected power. Filled symbols: signal; empty symbols: idler; circles: anomalous dispersion regime (with $\lambda_{\text {inj }}=873 \mathrm{~nm}$ ); squares: normal dispersion regime (with $\lambda_{\text {inj }}=$ $825 \mathrm{~nm}$ ); upward and downward triangles: internal and external maxima, respectively, in the transition regime (with $\lambda_{\text {inj }}=830 \mathrm{~nm}$ ). The dashed and solid lines correspond to the simulation results with an interaction length $L_{\mathrm{FWM}}=L_{\text {eff }}=0.9 L_{\text {fiber }}$ and $L_{\mathrm{FWM}}=0.8 L_{\text {eff }}=$ $0.7 L_{\text {fiber }}$, respectively.

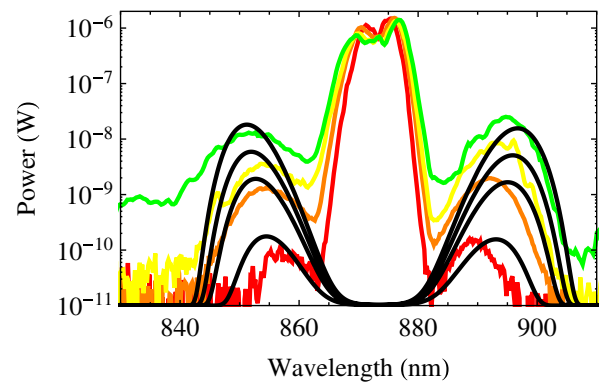

Fig. 11. Measured spectra for $\lambda_{\text {inj }}=873 \mathrm{~nm}$ and with an OSA spectral resolution of $2 \mathrm{~nm}$. Black solid lines correspond to the simulation results [based on Eqs. (7) and (11)] with $L_{\mathrm{FWM}}=0.8 L_{\text {eff }}=0.7 L_{\text {fiber }}$ and pump peak powers equal to the injected peak powers: $\{175$; $210 ; 225 ; 240\} \mathrm{W}$.

(2) The right maximum power level, provided an interaction length $L_{\text {FWM }}$ reduced by a factor 0.8 from the effective length $L_{\text {eff }}$ (i.e., by a factor 0.7 from the physical length $L_{\text {fiber }}$ ), thus showing that the temporal walk-off effect actually has a small but real influence.

(3) And last but not least, a sideband spectral width consistent with the experimental results, showing that SPMinduced spectral broadening of the injected pulses has no major influence (since our model is based on the quasimonochromatic field approximation), at least in a low-power regime.

The problem is that the other data series in Fig. 10 are not aligned (as they should be, however) on the same straight line as the experimental results obtained in the anomalous dispersion regime. Interestingly, this result is consistent with our interpretation of the phenomenon. Indeed, in the anomalous dispersion regime, the total injected power is used as a pump for the degenerate FWM process. But, by contrast, it is not the case in the normal dispersion regime, in which only the part of the spectrum that has crossed beyond $830 \mathrm{~nm}$ typically (thanks to SPM-induced spectral broadening) is effectively used. Plotting the evolution of $P_{s, i}$ as a function of this useful power (rather than the total injected power) should lead to a common alignment of all data series on the same straight line.

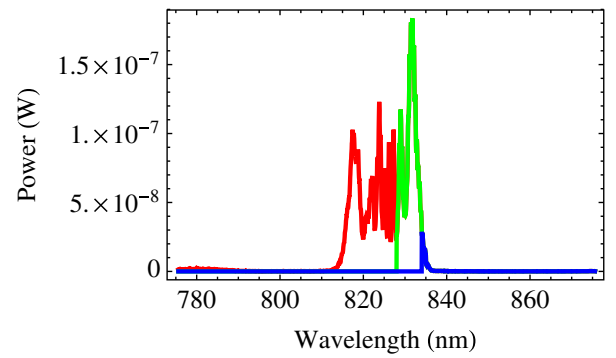

Fig. 12. Example of SPM-broadened spectrum recorded in linear scale and with an OSA resolution of $0.2 \mathrm{~nm} . P_{\text {inj }}$ is proportional to the integral of the entire spectrum (red); $P_{\text {useful } 828 \mathrm{~nm}}$ and $P_{\text {useful, } 834 \mathrm{~nm}}$ are proportional to the integrals of the green part and the blue part, respectively (with the same proportionality factor).

To determine the value of the useful power, we record SPM-broadened spectra with a spectral resolution of $0.2 \mathrm{~nm}$ on the OSA and with a linear power scale (see Fig. 12). The total injected peak power $P_{\text {inj }}$ is proportional to the integral of the entire associated spectrum, whereas the useful peak power is proportional (with the same proportionality factor) to the integral of this spectrum above a cutoff wavelength $\lambda_{c}$ only. The value $\lambda_{c}$ has to be precisely determined, knowing that it is around $830 \mathrm{~nm}$.

To determine the precise value of $\lambda_{c}$, we record a series of spectra (as described in the previous paragraph) keeping $\lambda_{\text {inj }}=825 \mathrm{~nm}$ and varying $P_{\text {inj }}$ from 330 to $530 \mathrm{~W}$. For each of these spectra, we try out several cutoff wavelengths (from 815 to $835 \mathrm{~nm}$ with an increment of $1 \mathrm{~nm}$ ), and we calculate the useful powers for each of the spectra and each of the tested cutoff wavelengths. In what follows, we call them $P_{\text {useful }, \lambda_{c}}$. We choose 815 and $835 \mathrm{~nm}$ to be the two extreme values of tried cutoff wavelengths because (1) the correction made with $\lambda_{c}=815 \mathrm{~nm}$ is expected to be weak, since $P_{\text {useful }, 815 \mathrm{~nm}}$ will remain close to $P_{\text {inj }}$, and (2) by contrast $P_{\text {useful, } 835 \mathrm{~nm}}$ will be equal to zero (for the lowest values of $\left.P_{\text {inj }}\right)$ or close to zero since a very small part of the spectra can reach wavelengths higher than $835 \mathrm{~nm}$ (see Fig. 12).

Then we plot the red data series of Fig. 10 as a function of all the $P_{\text {useful, } \lambda_{c}}$ with $\lambda_{c}$ varying from 815 to $8 \overline{35} \mathrm{~nm}$, and we find that the only value of $\lambda_{c}$ leading to an alignment of these red data series on the theoretical blue straight line of Fig. 10 is around $828 \mathrm{~nm}$. To further confirm this result, we then calculate $P_{\text {useful, } 828 \mathrm{~nm}}$ for $\lambda_{\text {inj }}=830 \mathrm{~nm}$ and $P_{\text {inj }}$ varying from 230 to $510 \mathrm{~W}$ (with still the same procedure, recording SPMbroadened spectra with a spectral resolution of $0.2 \mathrm{~nm}$ on the OSA), and we plot the green and black data series (associated with internal and external maxima, respectively) of Fig. 10 as a function of these $P_{\text {useful, } 828 \mathrm{~nm}}$. We also retrieved the alignment of these data series on the theoretical blue straight line (see Fig. 13, which shows the common alignment of all the data series on this blue straight line). Thus we can conclude that the correct value of $\lambda_{c}$ is about $828 \mathrm{~nm}$ (with a precision of about $2 \mathrm{~nm}$ ).

It is important to note that the fact that this correction provides a common alignment for all the data series (i.e., that there actually exists a unique cutoff wavelength, which is valid for all the values of $\lambda_{\text {inj }}$ ) is a quantitative confirmation of the analysis we made based on our first study (when varying the injected wavelength). Indeed, this result confirms that an efficient FWM can be observed even if the phase-matching 


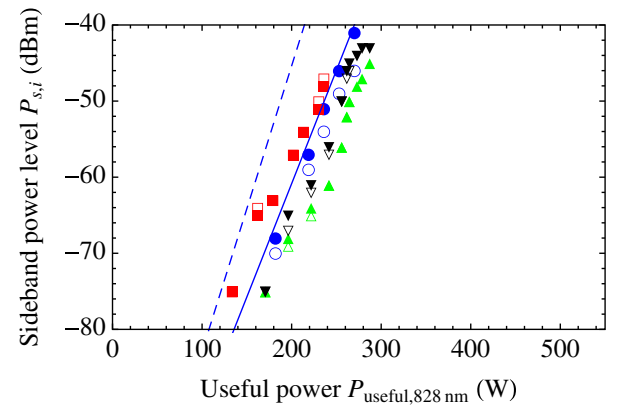

Fig. 13. Measured sideband power level plotted as a function of the "right" useful power $P_{\text {useful. } 828 \mathrm{~nm}}$. (See caption of Fig. 10.)

curve (which has been calculated neglecting the effect of SPM-induced spectral broadening) predicts that it should not occur because a part of the SPM-broadened injected spectrum falls beyond the vertical asymptote and thus intercept the phase-matching curve.

We also easily understand the origin of the frequency dissymmetry, since we can clearly see that $\lambda_{\text {source }}$ (which can be seen as the central wavelength of the useful spectrum, see Fig. 12) is necessarily higher than $\lambda_{\text {inj }}$ (central wavelength of the entire broadened spectrum).

\section{Discussion on the Origin of Double-Maximum Sideband Generation}

As illustrated in Fig. 9, spectra that are recorded in the transition regime II.b, exhibit two double-maximum sidebands (whereas there is only one maximum for each sideband generated in zones I and II.a). These two maxima are clearly distinguishable, particularly when $\lambda_{\text {inj }}$ ranges between 825 and $835 \mathrm{~nm}$ typically.

The exact origin of such a double-maximum sideband generation is not obvious. One avenue that could be explored involves the effect of temporal walk-off. The interaction length $L_{\text {FWM }}$ changes weakly with $\lambda_{\text {inj }}$ (at least on the range of $\lambda_{\text {inj }}$ that we study). However, these weak variations could be responsible for the presence of two local maxima (rather than a unique one) on the amplification gain curve as a function of wavelength, for a given pump wavelength [see Eq. (7)]. In a qualitative way, we can understand the problem as follows.

When $\lambda_{\text {inj }}$ moves away from the zero-dispersion wavelength of the fiber $\left(\lambda_{0}=834 \mathrm{~nm}\right)$, the GVM increases between the pump pulse and the generated pulses. In the anomalous dispersion regime, the signal and idler wavelengths are quite close to the pump wavelength, and thus GVD-induced temporal walk-off has a relatively weak impact (the interaction length is reduced by a factor of $\sim 0.8$ compared to the effective length of the fiber, which is already reduced by a factor of $\sim 0.9$ compared to the physical length of the fiber; thus we have $L_{\mathrm{FWM}} \sim 0.7 L_{\text {fiber }}$ ).

By contrast, when $\lambda_{\text {inj }}$ decreases from $\lambda_{0}$, the situation becomes more complex, with the apparition of two maxima in the FWM sidebands. We interpret this coexistence as a signature of the competition between the spectrally large FWM gain and the temporal walk-off.

The internal maxima correspond to a situation with low temporal walk-off as the frequencies stay close to the pump, but with a smaller gain as those frequencies are generated far from the maximum of the gain curve. The amplitude of the internal maxima goes lower as $\lambda_{\text {inj }}$ decreases further, since they are generated farther and farther from the gain maximum.

At the same time, the external maxima are generated with a high gain as they are closer to the gain maximum, which is enough to compensate for their larger temporal walk-off coming from a larger frequency separation from the pump. Paradoxically, those external maxima become less and less sensitive to the temporal walk-off, as they need (to be generated) a sufficiently large SPM-induced spectral broadening of the injected pulses to create the source wavelength. When $\lambda_{\text {inj }}$ decreases, the source wavelength is created closer and closer to the fiber output, and the remaining fiber length is no longer sufficient to generate a substantial temporal separation between the various frequency components. Thus the amplitude of the external maxima becomes larger and larger compared to the amplitude of the internal maxima (see Fig. 7).

\section{CONCLUSION}

We experimentally demonstrate that an efficient FWM process can be observed in the normal dispersion regime of a fiber, in contradiction with the prediction provided by the phasematching curve calculated from the GVD measurement of the fiber. We show that it is the result of the combination of two nonlinear phenomena: degenerate FWM and SPM. Indeed, whereas the phase-matching curve is usually plotted without taking into account the possibility of a spectral broadening of the injected pulses during their propagation inside the fiber, we can see that, under certain conditions, the decoupling between FWM and SPM-induced spectral broadening is not valid anymore.

One of the unexpected features of this phenomenon lies in its frequency-dissymmetric behavior. Of course, this frequency dissymmetry does not mean that the energy conservation is not satisfied, but only that the effective pump frequency $\omega_{\text {source }}$ is slightly different from the injected frequency $\omega_{\text {inj. }}$.

Such a frequency-dissymmetric FWM can be observed only if the phase-matching curve for degenerate FWM exhibits a vertical asymptote, i.e., if no efficient FWM process is expected when pumping below the zero-dispersion wavelength typically. As a result, it is worth noting that this phenomenon advantageously presents an extension of the spectral range on which an efficient FWM process can occur: instead of being restricted to injected pulse wavelengths higher than the zerodispersion wavelength $\lambda_{0}$ of the fiber, experimenters can work with lower wavelengths, providing the availability of a high enough peak power and/or interaction length to sufficiently broaden (by SPM) the injected pulse spectrum.

Our experimental investigations, combined with some simple analytical models, have allowed us to understand the origin of this surprisingly unexpected and frequencydissymmetric FWM process. Some more complete analytical and/or numerical models could allow us to confirm our analyses and moreover to clearly identify the origin of the doublemaximum sideband generation occurring in the transition regime. Such models should take into account FWM, SPM, Raman scattering, and, last but not least, propagation effects such as temporal walk-off. 


\section{ACKNOWLEDGMENTS}

This work is financially supported by the C'Nano Ile-deFrance "GENEPHY" project, and Margaux Barbier acknowledges the DGA for its financial support.

\section{REFERENCES}

1. M. Marhic, Fiber Optical Parametric Amplifiers, Oscillators and Related Devices (Cambridge University, 2012).

2. J. Dudley, G. Genty, and S. Coen, "Supercontinuum generation in photonic crystal fiber," Rev. Mod. Phys. 78, 1135-1184 (2006).

3. G. Agrawal, Applications of Nonlinear Fiber Optics (Academic, 2008).

4. J. Hansryd, P. Andrekson, M. Westlund, J. Li, and P.-O. Hedekvist, "Fiber-based optical parametric amplifiers and their applications,” IEEE J. Sel. Top. Quantum Electron. 8, 506-520 (2002).

5. L. Wang, C. Hong, and S. Friberg, "Generation of correlated photons via four-wave mixing in optical fibers," J. Opt. B 3, 346-352 (2001).

6. O. Alibart, J. Fulconis, G. Wong, S. Murdoch, W. Wadsworth, and J. Rarity, "Photon-pair generation using four-wave mixing in a microstructured fiber: theory versus experiment," New J. Phys. 8, 67 (2006)

7. J. Fan, A. Migdall, J. Chen, E. Goldschmidt, and A. Ling, "Microstructure fiber-based source of photonic entanglement," IEEE J. Sel. Top. Quantum Electron. 15, 1724-1732 (2009).

8. P. Russel, "Photonic-crystal fibers," J. Lightwave Technol. 24, 4729-4749 (2006).
9. V. Finazzi, T. Monro, and D. Richardson, "Small-core silica holey fibers: nonlinearity and confinement loss trade-offs,” J. Opt. Soc. Am. B 20, 1427-1436 (2003).

10. S. Coen, A. L. Chau, R. Leonhardt, J. Harvey, J. Knight, W. Wadsworth, and P. Russel, "Supercontinuum generation by stimulated Raman scattering and parametric four-wave mixing in photonic crystal fibers," J. Opt. Soc. Am. B 19, 753-764 (2002).

11. P. Roberts, P. Mangan, H. Sabert, F. Couny, T. Birks, J. Knight and P. Russel, "Control of dispersion in photonic crystal fibers," J. Opt. Fiber. Commun. Rep. 2, 435-461 (2005).

12. F. Benabid, J. Knight, G. Antonopoulos, and P. Russel, "Stimulated Raman scattering in hydrogen-filled hollow-core photonic crystal fiber," Science 298, 399-402 (2002).

13. S. Lebrun, P. Delaye, R. Frey, and G. Roosen, "High-efficiency single-mode Raman generation in a liquid-filled photonic bandgap fiber," Opt. Lett. 32, 337-339 (2007).

14. G. Agrawal, Nonlinear Fiber Optics (Academic, 2007).

15. M.-C. Phan Huy, A. Baron, S. Lebrun, R. Frey, and P. Delaye, "Characterization of self-phase modulation in liquid-filled hollow core photonic bandgap fibers," J. Opt. Soc. Am. B 27, 1886-1893 (2010).

16. A. Bogris, D. Syvridis, P. Kylemark, and P. Andrekson, "Noise characteristics of dual-pump fiber-optic parametric amplifiers," J. Lightwave Technol. 23, 2788-2795 (2005).

17. A. Weiner, Ultrafast Optics (Wiley, 2009).

18. K. Saitoh and M. Koshiba, "Empirical relations for simple design of photonic crystal fibers," Opt. Express 13, 267-274 (2005).

19. M. Koshiba and K. Saitoh, "Applicability of classical optical fiber theories to holey fibers,” Opt. Lett. 29, 1739-1741 (2004). 